\title{
ON A CLASS OF INNER FUNCTIONS IN A HALF-SPACE
}

\author{
F.A. SHAMOYAN \\ Dedicated to the memory of Professor \\ Igor' Fedorovich Krasichkov-Ternovskii
}

\begin{abstract}
In the paper we obtain necessary and sufficient conditions for the weight vector function, under which a given inner function is weakly invertible in the weighted space of holomorphic functions in a tubular domain.
\end{abstract}

Keywords: weak invertibility, weighted spaces, tubular domain.

Mathematics Subject Classification: 32A36, 32A37, 47A16, 47A15, 42B35

\section{INTRODUCTION}

Let $\mathbb{C}^{n}$ be an $n$-dimensional complex space, $G$ be a domain in $\mathbb{C}^{n}, H(G)$ be the set of analytic in $G$ functions, $H^{\infty}(G)$ be the set of all bounded analytic in $G$ functions. We suppose that $X$ is a some topological subspace of space $H(G)$, in which $H^{\infty}(G)$ is a dense set, operators $S_{z}(f)=f(z), z \in G$, and $M_{\psi}(f)=\psi f, \psi \in H^{\infty}, f \in X$, are bounded operators in $X$.

Definition 1. Let $f \in X$ and there exists a sequence $f_{m} \in H^{\infty}(G)$ such that $\lim _{m \rightarrow+\infty} f_{m} f=1$ in the sense of the topology in space $X$. Then function $f$ is called weakly invertible in space $X$.

Thus, $f$ is weakly invertible $X$ if set $H^{\infty}(G) f$ is everywhere dense in space $X$.

We note that the issue on weak invertibility in particular functional space are related with a wide class of problems in several fields, from the theory of differential operators and their generalization till abstract harmonic analysis [1].

In the one-dimensional case the weak invertibility was studied in the classical work by M.V. Keldysh [2], where it was established that there exists a function $f \in H^{\infty}(D), f(z) \neq 0$, $z \in D=\left\{z \in \mathbb{C}^{1}:|z|<1\right\}$ not weakly invertible in the Bergman space

$$
A^{p}(D)=\left\{f \in H(D):\|f\|_{A^{p}(D)}=\left(\int_{D}|f(z)|^{p} d m_{2}(z)\right)^{\frac{1}{p}}<+\infty\right\},
$$

where $m_{2}$ is the planar Lebesgue measure. In these constructions an important role was played by the inner function $S(z)=\exp \left(-\frac{1+z}{1-z}\right), z \in D$.

F.A. Shamoyan, On a Class of inner FunCtions in A HALF-SPACE.

(C) Shamoyan F.A. 2015.

The work was supported by the Ministery of Education and Science of Russian Federation (project no. 1.1704.2014K) and the Russian Foundation of Basic Researches (project no. 13-01-97508).

Submitted October 12, 2015. 
In works by A. Berling [3] and N. Nikolskii [4] the weak invertibility of function $S$ in the weighted space

$$
A_{\varphi}^{p}=\left\{f \in H(D):\|f\|_{A_{\varphi}^{p}}=\left(\int_{D}|f(z)|^{p} \exp \left(-\varphi\left(\frac{1}{1-|z|}\right)\right) d m_{2}(z)\right)^{\frac{1}{p}}<+\infty\right\}
$$

was studied.

Under certain restrictions for regularity of the growth of $\varphi$, it was established in these works that the weak invertibility of function $S$ in space $A_{\varphi}^{p}, 1 \leqslant p<+\infty$, is equivalent to

$$
\int_{1}^{+\infty}\left(\frac{\varphi(x)}{x^{3}}\right)^{\frac{1}{2}} d x=+\infty
$$

Taking into consideration that function $S$ is analytic everywhere except the point $z=1$, the author and his $\mathrm{PhD}$ student I. Gevorkyan in [5] studied the weak invertibility of function $S$ in the space

$$
\begin{aligned}
\tilde{A}_{\varphi}^{p}=\{f \in H(D): \\
\\
\left.\quad\|f\|_{\tilde{A}_{\varphi}^{p}}=\left(\int_{D}|f(z)|^{p} \exp \left(-\varphi\left(\frac{1}{|1-z|}\right)\right) d m_{2}(z)\right)^{\frac{1}{p}}<+\infty, \quad 1 \leqslant p<+\infty\right\} .
\end{aligned}
$$

It was established in [5] that as opposed to (1), the criterion of the weak invertibility of function $S$ in $\tilde{A}_{\varphi}^{p}, 0<p \leqslant+\infty$, reads as

$$
\int_{1}^{+\infty} \frac{\varphi(x)}{x^{2}} d x=+\infty
$$

It is obvious that condition (2) implies (1), but the converse is false.

In a recent work [6], a new proof of the above results by A. Berling, N.Nikolskii and I. Gevorkyan-F. Shamoyan was proposed for the case $p=2$; the proof was based on the well known corona theorem.

In the present work we study the issues of this type in multi-dimensional (tubular) domains.

\section{MAin RESUlts AND PROOF OF AUXILIARY STATEMENTS}

To present the main results of the work we introduce the following notations.

Let $P(x)=\left(p_{1}\left(x_{1}\right), \ldots, p_{n}\left(x_{n}\right)\right), x=\left(x_{1}, \ldots, x_{n}\right)$, be a vector function defined on $\mathbb{R}_{+}^{n}=$ $\left\{x=\left(x_{1}, \ldots, x_{n}\right) \in \mathbb{R}^{n}, x_{j}>0, j=\overline{1, n}\right\}, \mathbb{C}_{+}^{n}$ be the tubular domain with the basis $\mathbb{R}_{+}^{n}$, i.e., $\mathbb{C}_{+}^{n}=\left\{z=\left(z_{1}, \ldots, z_{n}\right) \in \mathbb{C}^{n}:\left(\operatorname{Im} z_{1}, \ldots, \operatorname{Im} z_{n}\right) \in \mathbb{R}_{+}^{n}\right\}$.

Let

$$
A_{P}^{q}\left(\mathbb{C}_{+}^{n}\right)=\left\{f \in H\left(\mathbb{C}_{+}^{n}\right):\|f\|_{A_{P}^{q}}=\left(\int_{\mathbb{C}_{+}^{n}}|f(z)|^{p} \exp (-P(|z|)) d m_{2 n}(z)\right)^{\frac{1}{q}}<+\infty\right\},
$$

where $z=\left(z_{1}, \ldots, z_{n}\right), \exp (-P(|z|)):=\prod_{j=1}^{n} \exp \left(-p_{j}\left(\left|z_{j}\right|\right)\right) ; d m_{2 n}$ is the $2 n$-dimensional Lebesgue measure in $\mathbb{C}_{+}^{n}$. 
In what follows we assume that $p_{j}(x)=\int_{1}^{x} \frac{\omega_{j}(t)}{t} d t, j=\overline{1, n}$, where $\omega_{j}$ are defined on $\mathbb{R}_{+}:=\mathbb{R}_{+}^{1}$, and $\omega_{j}(t) \uparrow^{+\infty}(t \rightarrow+\infty), 1 \leqslant j \leqslant n$. Such functions will be called weights, while vectors functions $P=\left(p_{1}, \ldots, p_{n}\right)$ will be called weight vector function. The set of all weight vector functions is denoted by $\Omega$.

The main results of the paper are the two following statements.

Theorem 1. Let $a=\left(a_{1}, \ldots, a_{n}\right) \in \mathbb{R}_{+}^{n}, z=\left(z_{1}, \ldots, z_{n}\right) \in \mathbb{C}_{+}^{n}, a z=\sum_{j=1}^{n} a_{j} z_{j}, S_{a}(z)=$ $\exp \left(i \sum_{j=1}^{n} a_{j} z_{j}\right), z=\left(z_{1}, \ldots, z_{n}\right) \in \mathbb{C}_{+}^{n}, P=\left(p_{1}, \ldots, p_{n}\right) \in \Omega$. Then

1) the following statements are equivalent:

a) function $S_{a}$ is weakly invertible in space $A_{P}^{q}$ for some $q=q_{0}, 1 \leqslant q_{0}<+\infty$;

b) $S_{a}$ is weakly invertible in space $A_{P}^{q}$ for all $0<q<+\infty$;

c)

$$
\int_{1}^{+\infty} \frac{\mathrm{p}_{j}(t)}{t^{2}} d t=+\infty, j=\overline{1, n}
$$

2) If at least one of the integrals in (3) converges, then function $S_{a}$ is not weakly invertible in each space $A_{P}^{q}, 0<q<+\infty$.

Theorem 2. Let $P=\left(p_{1}, \ldots, p_{n}\right)$ be a vector function in $\Omega, f \in H^{\infty}\left(\mathbb{C}_{-\eta}^{n}\right)$, where $\mathbb{C}_{-\eta}^{n}=$ $\left\{z=\left(z_{1}, \ldots, z_{n}\right): \operatorname{Im} z_{j}>-\eta, j=\overline{1, n}\right\}, f(z) \neq 0, z \in \mathbb{C}_{-\eta}^{n}, 0<s<1$.

Let $M_{m}=\sup _{z \in \mathbb{C}_{+}^{n}}\left\{|\ln f(z)|^{m} \exp (-s P(|z|))\right\}$, where the principal branch of the logarithm is fixed. If

$$
\sum_{m=1}^{\infty} \frac{1}{\sqrt[m]{M_{m}}}=+\infty
$$

then function $f$ is weakly invertible in space $A_{P}^{q}\left(\mathbb{C}_{+}^{n}\right)$ for all $0<q<+\infty$.

Remark 1. We note that the conditions $f \in H^{\infty}\left(\mathbb{C}_{+}^{n}\right)$ and $f(z) \neq 0, z \in \mathbb{C}_{+}^{n}$, are not sufficient for the weak invertibility of function $f$ in space $A_{P}^{q}\left(\mathbb{C}_{+}^{n}\right)$.

Indeed, in view of the results of work [8], it is easy to establish that the functions $f_{a}(z)=$ $\exp \left(-\sum_{j=1}^{n} \frac{i c_{j}}{z_{j}-a_{j}}\right), z=\left(z_{1}, \ldots, z_{n}\right) \in \mathbb{C}_{+}^{n}, a=\left(a_{1}, \ldots, a_{n}\right) \in \mathbb{R}^{n}, c=\left(c_{1}, \ldots, c_{n}\right) \in \mathbb{R}_{+}^{n}$, are not weakly invertible in space $A_{P}^{q}\left(\mathbb{C}_{+}^{n}\right)$.

Remark 2. If series (4) diverges, and function $f$ coincides with function $S_{a}$, then it follows from Theorem 1 that function $f$ is not weakly invertible in space $A_{P}^{q}\left(\mathbb{C}_{+}^{n}\right)$ for each $q>0$, since the convergence of series (4) is equivalent to the convergence of integrals (3) (see [12]).

Before proving Theorems 1 and 2, we provide the following auxiliary statements.

Let $k=\left(k_{1}, \ldots, k_{n}\right)$ be a permutation of numbers $(1,2, \ldots, n), n \in \mathbb{N}, 1 \leqslant m \leqslant n$. Then the vector with the coordinates $\left(k_{1}, \ldots, k_{m}\right)$ is called a tuple of order $m$. The set of all tuples of order $m$ is denoted by $K_{m}$. It is clear that if $1 \leqslant r, m \leqslant n$, then the identity $\left(k_{1}, \ldots, k_{r}\right)=\left(s_{1}, \ldots, s_{m}\right)$ holds true if and only if $r=m, s_{i}=k_{i}, i=\overline{1, m}$.

Lemma 1. Let $f \in H\left(\mathbb{C}_{+}^{n}\right), k=\left(k_{1}, \ldots, k_{m}\right) \in K_{m}, \widetilde{z}=\left(\widetilde{z_{1}}, \ldots, \widetilde{z_{n}}\right) \in \mathbb{C}_{+}^{n}$, and $\widetilde{z_{j}}=z_{k_{j}}$, if $j=k_{j}$ for some $k_{j} \in K_{m}$, and $\widetilde{z_{j}}=i$, if $j \neq k_{j}, j=\overline{1, n}$. 
Suppose that $P=\left(p_{1}, \ldots, p_{n}\right)$ is a weight vector function, $P \in \Omega$. If $0<s<+\infty$, then the estimate

$$
|f(\widetilde{z})|^{s} \exp (-P(2|\widetilde{z}|)) \leqslant \frac{c_{0}(s)}{\prod_{j=1}^{m} y_{k_{j}}^{2}} \int_{\widetilde{U}^{n}(\widetilde{z})}|f(\zeta)|^{s} \exp (-P(|\zeta|)) d m_{2 n}(\zeta)
$$

holds true, where $\widetilde{U}^{n}(\widetilde{z})=\left\{\zeta=\left(\zeta_{1}, \ldots, \zeta_{n}\right) \in \mathbb{C}_{+}^{n}:\left|\zeta_{j}-\widetilde{z}_{j}\right|<\frac{\operatorname{Im} \widetilde{z_{j}}}{2}, j=\overline{1, n}\right\}$.

Proof. Without loss of generality we can assume that $j=k_{j}, 1 \leqslant j \leqslant m$. Then

$$
\widetilde{U}^{n}(\widetilde{z})=\left\{\zeta=\left(\zeta_{1}, \ldots, \zeta_{n}\right):\left|\zeta_{j}-z_{j}\right|<\frac{y_{j}}{2}, 1 \leqslant j \leqslant m,\left|\zeta_{j}-i\right|<\frac{1}{2}, m+1 \leqslant j \leqslant n\right\} .
$$

Taking into consideration the $n$-subharmonicity of the function $|f(\zeta)|^{s}, \zeta \in \mathbb{C}_{+}^{n}$, we obtain

$$
|f(\widetilde{z})|^{s} \leqslant \frac{2^{2 m}}{\pi^{n} \prod_{j=1}^{m} y_{j}^{2}} \int_{\widetilde{U}^{n}(\widetilde{z})}|f(\zeta)|^{s} d m_{2 n}(\zeta)
$$

where $\widetilde{z}=\left(z_{1}, \ldots, z_{m}, i, \ldots, i\right)$. We note that if $\zeta \in \widetilde{U}^{n}, \zeta=\left(\zeta_{1}, \ldots, \zeta_{n}\right)$, then $\left|z_{j}-\zeta_{j}\right|<\frac{y_{j}}{2}$, $z_{j}=x_{j}+i y_{j}, j=\overline{1, m}$, and $\left|i-\zeta_{j}\right|<\frac{1}{2}$ if $j=\overline{m+1, n}$. Hence,

$$
\frac{\left|z_{j}\right|}{2} \leqslant\left|z_{j}\right|-\frac{\left|y_{j}\right|}{2} \leqslant\left|\zeta_{j}\right| \leqslant\left|z_{j}\right|+\frac{\left|y_{j}\right|}{2} \leqslant \frac{3}{2}\left|z_{j}\right|, \quad j=\overline{1, m} ; \quad \frac{1}{2} \leqslant\left|\zeta_{j}\right| \leqslant \frac{3}{2}, \quad j=\overline{m+1, n} .
$$

Therefore,

$$
\begin{aligned}
\exp \left(-p_{j}\left(\frac{3}{2}\left|z_{j}\right|\right)\right) & \leqslant \exp \left(-p_{j}\left(\left|\zeta_{j}\right|\right)\right) \\
& \leqslant \exp \left(-p_{j}\left(\frac{\left|z_{j}\right|}{2}\right)\right), \zeta=\left(\zeta_{1}, \ldots, \zeta_{n}\right) \in \widetilde{U}^{n}(\widetilde{z}), \quad j=\overline{1, n}
\end{aligned}
$$

Employing estimates (6), (7), we arrive at the inequality

$$
\begin{aligned}
|f(\widetilde{z})|^{s} \exp \left(-p_{j}\left(\frac{3}{2}|\widetilde{z}|\right)\right) & =\left|f\left(z_{1}, \ldots, z_{m}, i, \ldots, i\right)\right|^{s} \exp \left(-\sum_{j=1}^{m} p_{j}\left(\frac{3}{2}\left|z_{j}\right|\right)\right) \\
& \leqslant \frac{2^{2 m}}{\pi^{n} \prod_{j=1}^{m} y_{j}^{2}} \int|f(\zeta)|^{s} \exp -(P(|\zeta|)) d m_{2 n}(\zeta) \\
& \leqslant \frac{C(m, n)}{\prod_{j=1}^{m} y_{j}^{2}} \int_{\mathbb{C}_{+}^{n}}|f(\zeta)|^{s} \exp (-P(|\zeta|)) d m_{2 n}(\zeta) .
\end{aligned}
$$

The proof is complete.

The next statement was proved in the work by M.M. Dzhrbashyan [9], see also [10].

Lemma 2. Let $P=\left(p_{1}, \ldots, p_{n}\right)$ be a weight vector function, $1 \leqslant q<+\infty$. Then the following statements are equivalent:

1) the set of all algebraic polynomials of $\left(z_{1}, \ldots, z_{n}\right)$ is an everywhere dense in $A_{P}^{q}\left(\mathbb{C}_{+}\right)$set;

2) statements (3) of Theorem 1 hold true and if one of the integrals in (3) diverges, the set of the polynomials is not dense in space $A_{P}^{q}\left(\mathbb{C}_{+}^{n}\right)$ for arbitrary $0<q<+\infty$. 
Proof. Let $1 \leqslant q<+\infty$. We prove Lemma for $n=2$, for other $n$ the main milestones of the proof are same.

Let

$$
L_{P}^{q^{\prime}}\left(\mathbb{C}_{+}^{2}\right):=\left\{f \in S\left(\mathbb{C}_{+}^{n}\right):\left(\int_{\mathbb{C}_{+}^{2}}|f(\zeta)|^{q^{\prime}} \exp (-P(|\zeta|)) d m_{4}(\zeta)\right)^{\frac{1}{q^{\prime}}}<+\infty\right\}
$$

where $S$ is the set of all measurable on $\mathbb{C}_{+}^{n}$ functions, and $q^{\prime}=\frac{q}{q-1}$. Suppose that $g \in L_{P}^{q^{\prime}}\left(\mathbb{C}_{+}^{2}\right)$ is such that

$$
\int_{\mathbb{C}_{+}^{2}} g\left(\zeta_{1}, \zeta_{2}\right) \zeta_{1}^{k_{1}} \zeta_{2}^{k_{2}} e^{-p_{1}\left(\left|\zeta_{1}\right|\right)-p_{2}\left(\left|\zeta_{2}\right|\right)} d m_{4}\left(\zeta_{1}, \zeta_{2}\right)=0, k=\left(k_{1}, k_{2}\right) \in \mathbb{Z}_{+}^{2}
$$

Let us prove that

$$
\int_{\mathbb{C}_{+}^{2}} g\left(\zeta_{1}, \zeta_{2}\right) f\left(\zeta_{1}, \zeta_{2}\right) e^{-p_{1}\left(\left|\zeta_{1}\right|\right)-p_{2}\left(\left|\zeta_{2}\right|\right)} d m_{4}\left(\zeta_{1}, \zeta_{2}\right)=0
$$

for each $f \in A_{P}^{q}\left(\mathbb{C}_{+}^{2}\right)$.

Let $\widetilde{g}\left(\zeta_{1}\right)=\int_{\mathbb{C}_{+}} g\left(\zeta_{1}, \zeta_{2}\right) \exp \left(-p_{2}\left(\left|\zeta_{2}\right|\right)\right) d m_{2}\left(\zeta_{2}\right)$. It is obvious that $\widetilde{g}\left(\zeta_{1}\right)$ is an almost everywhere finite function. Let us prove that $\widetilde{g}\left(\zeta_{1}\right) \in L_{p_{1}}^{q^{\prime}}\left(\mathbb{C}_{+}\right)$. By the Hölder inequality we have

$$
\begin{aligned}
& \int_{\mathbb{C}_{+}}\left|\widetilde{g}\left(\zeta_{1}\right)\right|^{q^{\prime}} e^{-p_{1}\left(\left|\zeta_{1}\right|\right)} d m_{2}\left(\zeta_{1}\right)=\int_{\mathbb{C}_{+}}\left(\int_{\mathbb{C}_{+}}\left|g\left(\zeta_{1}, \zeta_{2}\right)\right| e^{-p_{2}\left(\left|\zeta_{2}\right|\right)} d m_{2}\left(\zeta_{2}\right)\right)^{q^{\prime}} e^{-p_{1}\left(\left|\zeta_{1}\right|\right)} d m_{2}\left(\zeta_{1}\right) \\
& \leqslant \int_{\mathbb{C}_{+}^{2}}\left|g\left(\zeta_{1}, \zeta_{2}\right)\right|^{q^{\prime}} e^{-p_{2}\left(\left|\zeta_{2}\right|\right)} e^{-p_{1}\left(\left|\zeta_{1}\right|\right)} d m_{4}\left(\zeta_{1}, \zeta_{2}\right)\left(\int_{\mathbf{C}_{+}} e^{-p_{2}\left(\left|\zeta_{2}\right|\right)} d m_{2}\left(\zeta_{2}\right)\right)^{\frac{q^{\prime}}{q}} \\
& \leqslant \text { const } \int_{\mathbb{C}_{+}}\left|g\left(\zeta_{1}\right)\right|^{q^{\prime}} e^{-p_{1}\left(\left|\zeta_{1}\right|\right)} d m_{2}\left(\zeta_{1}\right)<+\infty .
\end{aligned}
$$

Therefore, by M.M. Dzrbashyan theorem (see [9]),

$$
\int_{\mathbb{C}_{+}} \widetilde{g}\left(\zeta_{1}\right) f\left(\zeta_{1}\right) e^{-p_{1}\left(\left|\zeta_{1}\right|\right)} d m_{2}\left(\zeta_{1}\right)=0
$$

for an arbitrary $f \in A_{P_{1}}^{q}\left(\mathbb{C}_{+}\right)$.

Exactly in the same way one can prove that if $f \in A_{P_{1}}^{q}\left(\mathbb{C}_{+}^{2}\right)$, then the function $\tilde{f}\left(\zeta_{1}\right)=$ $\int_{\mathbb{C}_{+}} f\left(\zeta_{1}, \zeta_{2}\right) \exp \left(-p_{2}\left(\left|\zeta_{2}\right|\right)\right) d m_{2}\left(\zeta_{2}\right)$ belongs to class $A_{P_{1}}^{q}\left(\mathbb{C}_{+}\right)$. Hence, applying M.M. Dzrbashyan theorem, we obtain that

$$
\int_{\mathbb{C}_{+}} \tilde{g}\left(\zeta_{1}\right) \widetilde{f}\left(\zeta_{1}\right) d m_{2}\left(\zeta_{1}\right)=0
$$


i.e.,

$$
\int_{\mathbb{C}_{+}^{2}} g\left(\zeta_{1}, \zeta_{2}\right) f\left(\zeta_{1}, \zeta_{2}\right) \exp \left(-p_{1}\left(\left|\zeta_{1}\right|\right)\right) \exp \left(-p_{2}\left(\left|\zeta_{2}\right|\right)\right) d m_{4}\left(\zeta_{1}, \zeta_{2}\right)=0 .
$$

This identity and Hahn-Banach theorem imply the first part of the lemma.

We proceed to the proof of the second part. It follows from Lemma 1 that if the polynomials are dense in $A_{P}^{q}\left(\mathbb{C}_{+}^{n}\right)$, then there exists a sequence of polynomials $\tilde{P}_{m}(z)=\sum_{k=1}^{m} a_{k}^{(m)} z^{k}, z \in \mathbb{C}_{+}$ such that $\max _{z \in \mathbb{C}_{+}}\left\{\left|\tilde{P}_{m}(z)-f(z)\right| \exp \left(-p_{j}(|z|)\right)\right\}=0,1 \leqslant j \leqslant n$, for each $f \in A_{P_{j}}^{\infty}\left(\mathbb{C}_{+}\right) 0$. Then M.M. Dzhrbashyan theorem impliesL $\int_{1}^{+\infty} \frac{p_{j}(t)}{t^{2}} d t=+\infty$ (see [9], [10]).

The next lemma was proved in work [11].

Lemma 3. Let $p$ be a weight function such that

$$
\int_{0}^{+\infty} \frac{\mathrm{p}(t)}{1+t^{2}} d t<+\infty
$$

Suppose that $G$ is the external function in the half-plane $\mathbb{C}_{+}$readings as

$$
G(z)=\exp \left(-\frac{4 i}{\pi} \int_{-\infty}^{+\infty} \frac{t z+1}{t-z} \frac{p(t)}{1+t^{2}} d t\right), \quad z \in \mathbb{C}_{+} .
$$

Then there exists a positive number $c$ such that

$$
\exp (-c p(3|z|)) \leqslant|G(z)| \leqslant \exp (-p(|z|)), \quad z \in \mathbb{C}_{+} .
$$

\section{Proof of the MAin Results}

Proof of Theorem 1. We begin with the first statement c) $\Rightarrow$ b).

Let $1 \leqslant q<+\infty, a=\left(a_{1}, \ldots, a_{n}\right), a_{j}>0, j=\overline{1, n}$. We denote by $E_{q}\left(S_{a}\right)$ the closure of set $H^{\infty}\left(\mathbb{C}_{+}^{n}\right) S_{a}$ in space $A_{P}^{q}\left(\mathbb{C}_{+}^{n}\right)$. To prove the desired statement, it is sufficient to show that $1 \in E_{q}\left(S_{a}\right)$.

Let $\Phi$ be a linear continuous functional orthogonal to $E_{q}\left(S_{a}\right)$. Let us prove that $\Phi(1)=0$. We suppose that $\Phi$ is generated by some function $\Psi \in L_{P}^{q^{\prime}}\left(\mathbb{C}_{+}^{n}\right)$, where $\frac{1}{q}+\frac{1}{q^{\prime}}=1$, then

$$
\Phi\left(S_{a} F\right)=\int_{\mathbb{C}_{+}^{n}} e^{i a z} F(z) \Psi(z) e^{-P(|z|)} d m_{2 n}(z)=0,
$$

for each $F \in H^{\infty}\left(\mathbb{C}_{+}^{n}\right)$ as well as for $F\left(z_{1}, \ldots, z_{n}\right)=z_{1}^{m_{1}} \ldots z_{n}^{m_{n}}, m=\left(m_{1}, \ldots, m_{n}\right) \in \mathbb{Z}_{+}^{n}$.

Given $t \in[0,1]$, we let $e_{1}(t)=\int_{\mathbb{C}_{+}^{n}} e^{i a_{1} t z_{1}+i \widetilde{a} \widetilde{z}} \Psi(z) e^{-P(|z|)} d m_{2 n}(z)$, where $\widetilde{z}=\left(z_{2}, z_{3}, \ldots, z_{n}\right)$, $\widetilde{a}=\left(a_{2}, \ldots, a_{n}\right)$.

It is clear that

$$
e_{1}^{(m)}(t)=\int_{\mathbb{C}_{+}^{n}} e^{i a_{1} z_{1} t+i \widetilde{a} \widetilde{z}}\left(i a_{1} z_{1}\right)^{m} \Psi(z) e^{\left(-p_{1}\left(\left|z_{1}\right|\right)-\widetilde{P}(|z|)\right)} d m_{2 n}(z),
$$


where $\exp (-\widetilde{P}(|\widetilde{z}|))=\exp \left(-p_{2}\left(\left|z_{2}\right|\right) \ldots-p_{n}\left(\left|z_{n}\right|\right)\right)$. It is obvious that

$$
e_{1}^{(m)}(1)=0, \quad m \in \mathbb{Z}_{+} .
$$

Let us prove that function $e$ belongs to the quasi-analytic class on the segment $[0,1]$ (see [12]). Indeed, applying Hölder inequality, we have

$$
\begin{aligned}
\left|e_{1}^{(m)}(t)\right| \leqslant & \int_{\mathbb{C}_{+}^{n}} \exp \left(-p_{1}\left(\left|z_{1}\right|\right)\right)\left|a_{1}\right|^{m}\left|z_{1}\right|^{m}|\Psi(z)| \exp (-\widetilde{P}(|\widetilde{z}|)) d m_{2 n}(z) \\
\leqslant & \left|a_{1}\right|^{m}\left(\int_{\mathbb{C}_{+}} e^{\left(-p_{1}\left(\left|z_{1}\right|\right)\right)}\left|z_{1}\right|^{m q} d m_{2}\left(z_{1}\right)\right)^{\frac{1}{q}} \\
& \cdot\left(\int_{\mathbb{C}_{+}} e^{\left(-p_{1}\left(\left|z_{1}\right|\right)\right)}\left(\int_{\mathbb{C}_{+}^{n-1}}|\Psi(z)| e^{(-\widetilde{P}(|z|))} d m_{2 n-2}(z)\right)^{q^{\prime}} d m_{2}(z)\right)^{\frac{1}{q^{\prime}}} .
\end{aligned}
$$

Applying Hölder inequality once again, we arrive at the estimate

$$
\begin{aligned}
\left|e_{1}^{(m)}(t)\right| \leqslant & \left|a_{1}\right|^{m}\left(\int_{\mathbb{C}_{+}}\left|z_{1}\right|^{m q} e^{\left(-p_{1}\left(\left|z_{1}\right|\right)\right)} d m_{2}(z)\right)^{\frac{1}{q}} \\
& \left(\int_{\mathbb{C}_{+}} e^{\left(-p_{1}\left(\left|z_{1}\right|\right)\right)}\left(\int_{\mathbb{C}_{+}^{n-1}}|\Psi(z)|^{q^{\prime}} e^{(-\widetilde{P}(|z|))} d m_{2 n-2}(z)\right) d m_{2 n}(z)\right) \\
& \cdot\left(\int_{\mathbb{C}_{+}^{n-1}} e^{(-\widetilde{P}(|z|))} d m_{2 n-2}(z)\right)^{\frac{q^{\prime}}{q}} \\
\leqslant & C_{1}\left|a_{1}\right|^{m}\left(\int_{\mathbb{C}_{+}}\left|z_{1}\right|^{m q} e^{\left(-p_{1}\left(\left|z_{1}\right|\right)\right)} d m_{2}\left(z_{1}\right)\right)^{\frac{1}{q}}\left(\int_{\mathbb{C}_{+}^{n}}|\Psi(z)|^{q^{\prime}} e^{(-P(|z|))} d m_{2 n}(z)\right)^{\frac{1}{q^{\prime}}} .
\end{aligned}
$$

Let $\delta$ be an arbitrary positive number $\delta \in(0,1)$. Then the latter estimate yields

$$
\begin{aligned}
\left|e_{1}^{(m)}(t)\right| \leqslant C_{2}\left|a_{1}\right|^{m} \sup _{r>0}\left(r^{\bar{m}} e^{-\frac{\delta}{q} p_{1}(r)}\right)\left(\int_{\mathbf{C}_{+}} e^{-(1-\delta) p_{1}(|z|)} d m_{2}(z)\right)^{\frac{1}{q}} \\
=C_{2}\left|a_{1}\right|^{m} \sup _{r>0}\left(r^{\bar{m}} e^{-\frac{\delta}{q} p_{1}(r)}\right)\left(\int_{0}^{+\infty} \int_{0}^{\pi} e^{-(1-\delta) p_{1}(|\rho|)} \rho d \rho d \varphi\right)^{\frac{1}{q}}=C_{3}\left|a_{1}\right|^{m} \sup _{r>0}\left(r^{\bar{m}} e^{-\frac{\delta}{q} p_{1}(r)}\right) .
\end{aligned}
$$

Thus, we finally obtain

$$
\left|e_{1}^{(m)}(t)\right| \leqslant C_{3}\left|a_{1}\right|^{m} M_{m}
$$

where $M_{m}=\sup _{r>0}\left(r^{\bar{m}} e^{-\frac{\delta}{q} p_{1}(r)}\right)$.

Now we employ Carleman-Ostrowski theorem (see [12]) on the quasi-analyticity of the class

$$
C^{\infty}\left(M_{m}\right)=\left\{\varphi \in C^{\infty}[0,1]:\left|\varphi^{(m)}(t)\right| \leqslant A^{m} M_{m}\right\}
$$


in accordance to which the criterion of the quasi-analyticity of class $C^{\infty}\left(M_{m}\right)$ is

$$
\int_{1}^{+\infty} \frac{\ln T(r)}{r^{2}} d r=+\infty
$$

where $T(r)=\sup _{r \geqslant 1} \frac{r^{m}}{M_{m}}($ see $[12])$.

But by M.M. Dzhrbashyan theorem (see [9], [10]), the convergence of the integral $\int_{1}^{+\infty} \frac{p_{1}(r)}{r^{2}} d r$ is equivalent to condition (15). Therefore, function $e(t)$ belongs to the quasi-analytic CarlemanOstrowsi class on the segment $[0,1]$. In view of condition (13) we have $e_{1}(t)=0, \forall t \in[0,1]$, i.e., $e_{1}(0)=0$. Hence,

$$
\int_{\mathbb{C}_{+}^{n}} e^{-i \tilde{a} \tilde{z}} \Psi(z) e^{-P(|z|)} d m_{2 n}(z)=0
$$

We let

$$
e_{2}(t)=\int_{\mathbb{C}_{+}^{n}} e^{-i a_{2} z_{2} t-i \widetilde{a} \widetilde{z}} \psi(z) e^{-p_{2}\left(\left|z_{2}\right|\right)-\tilde{P}(|z|)} d m_{2 n}(z),
$$

where $\widetilde{a}$ and $\widetilde{z}$ are introduced as above.

Reproducing the above arguments, we obtain $e_{2}(0)=0$. Repeating these arguments $n-1$ times, we obtain that

$$
\int_{\mathbb{C}_{+}^{n}} \Psi(z) e^{-P(|z|)} d m_{2 n}(z)=0
$$

i.e., $\Phi(1)=0$.

By Hahn-Banach theorem $1 \in E_{q}\left(S_{a}\right)$.

Thus, the implication $\mathrm{c}) \Rightarrow \mathrm{b}$ ) is proved under the condition $q \geqslant 1$. But since for an arbitrary $f \in H^{\infty}\left(\mathbb{C}_{+}^{n}\right), 0<q<1$,

$$
\left\|f S_{a}-1\right\|_{A_{P}^{q}\left(\mathbb{C}_{+}^{n}\right)} \leqslant\left\|f S_{a}-1\right\|_{A_{P}^{1}\left(\mathbb{C}_{+}^{n}\right)}\left(\int_{\mathbb{C}_{+}^{n}} e^{-P(|z|)} d m_{2 n}(z)\right)^{\frac{1-q}{q}},
$$

it completes the proof of this implication.

The implication $b) \Rightarrow c$ ) is obvious. This is why we have proved that $c) \Rightarrow b) \Rightarrow a$ ). To prove the first statement of the theorem, it remains to establish the implication a) $\Rightarrow c$ ).

It is obvious that the implication a) $\Rightarrow$ c) of the first statement is implied immediately by the second statement of the theorem. This is why we proceed to the proof of the second statement.

Suppose that there exists some $k=\left(k_{1}, \ldots, k_{m}\right) \in K_{m}$, such that

$$
\int_{1}^{+\infty} \frac{p_{k_{j}}(t)}{t^{2}} d t<+\infty, \quad j=\overline{1, m} .
$$

Without loss of generality we assume that $k_{j}=j, j=\overline{1, m}$. 
As in Lemma 3 , by means of function $p_{j}, j=\overline{1, m}$, we construct the set of external functions

$$
G_{j}(z)=\exp \left(\frac{-4 i}{\pi} \int_{-\infty}^{+\infty} \frac{t z+1}{t-z} \frac{p_{j}(3|t|)}{1+t^{2}} d t\right), \quad j=\overline{1, m} .
$$

We also let

$$
G(z)=\prod_{j=1}^{m} G_{j}\left(z_{j}\right)=\exp \left(\frac{-4 i}{\pi} \sum_{j=1}^{m}\left(\int_{-\infty}^{+\infty} \frac{t_{j} z_{j}+1}{t_{j}-z_{j}} \frac{p_{j}\left(3\left|t_{j}\right|\right)}{1+t_{j}^{2}} d t_{j}\right)\right), \quad z=\left(z_{1}, \ldots, z_{m}\right) \in \mathbb{C}_{+}^{m} .
$$

Employing Lemma 3, we obtain

$$
\exp \left(-c \sum_{j=1}^{m} p_{j}\left(9\left|z_{j}\right|\right)\right) \leqslant|G(z)| \leqslant \exp \left(-\sum_{j=1}^{m} p_{j}\left(3\left|z_{j}\right|\right)\right), \quad z=\left(z_{1}, \ldots, z_{m}\right) \in \mathbb{C}_{+}^{m}
$$

for some positive $c$.

Suppose that on the contrary to the second statement, there exists a sequence $\left\{f_{k}\right\}_{k=1}^{+\infty}$, $f_{k} \in H^{\infty}\left(\mathbb{C}_{+}^{n}\right)$, such that

$$
\lim _{k \rightarrow+\infty}\left\|f_{k} S_{a}-1\right\|_{A_{P}^{q}\left(\mathbb{C}_{+}^{n}\right)}=0 .
$$

Employing Lemma 1, we obtain

$$
\begin{aligned}
\mid f_{k}\left(z_{1}, \ldots, z_{m}, i, \ldots, i\right) & S_{a}\left(z_{1}, \ldots, z_{m}, i, \ldots, i\right)-\left.1\right|^{q} \exp \left(-\sum_{j=1}^{m} p_{j}\left(2\left|z_{j}\right|\right)\right) \\
\leqslant & \frac{c_{0}(q)}{\prod_{j=1}^{m} y_{j}^{2}}\left\|f_{k} S_{a}-1\right\|_{A_{P}^{q}\left(\mathrm{C}_{+}^{n}\right)}^{q}, \quad z=\left(z_{1}, \ldots, z_{m}, i, \ldots, i\right) \in \mathbb{C}_{+}^{n} .
\end{aligned}
$$

It follows immediately from estimates (16) and (18) that

$$
\begin{aligned}
& \left|f_{k}\left(z_{1}, \ldots, z_{m}, i, \ldots, i\right) S_{a}\left(z_{1}, \ldots, z_{m}, i, \ldots, i\right)-1\right|^{q}\left|G\left(z_{1}, \ldots, z_{m}\right)\right| \\
& \leqslant \frac{c(q)}{\prod_{j=1}^{m} y_{j}^{2}}\left\|f_{k} S_{a}-1\right\|_{A_{p}^{q}\left(\mathbb{C}_{+}^{n}\right)}^{q}, \quad z=\left(z_{1}, \ldots, z_{m}\right) \in \mathbb{C}_{+}^{n} .
\end{aligned}
$$

In particular, it follows immediately from estimate (19) that

$$
\begin{gathered}
\left|f_{k}\left(x_{1}+i, x_{2}+i, \ldots, x_{m}+i, i, \ldots, i\right) S_{a}\left(x_{1}+i, x_{2}+i, \ldots, x_{m}+i, i, \ldots, i\right)-1\right|^{q} \\
\cdot\left|G\left(x_{1}+i, x_{2}+i, \ldots, x_{m}+i\right)\right| \leqslant 1
\end{gathered}
$$

as $k \geqslant k_{0}$. Therefore,

$$
\begin{aligned}
\left|f_{k}\left(x_{1}+i, \ldots, x_{m}+i, i, \ldots, i\right)\right|^{q}\left|G\left(x_{1}+i, \ldots, x_{m}+i\right)\right|\left|S_{a}\left(x_{1}+i, \ldots, x_{m}+i, i, \ldots, i\right)\right|^{q} \\
\leqslant|G(x+i)|\left|f_{k}(\widetilde{x+i}) S_{a}(\widetilde{x+i})-1\right|^{q}+|G(x+i)| \leqslant 1+|G(x+i)|
\end{aligned}
$$

where $x+i=\left(x_{1}+i, x_{2}+i, \ldots, x_{m}+i, i, i \ldots, i\right) \in \mathbb{C}_{+}^{m}$.

It is obvious that estimate (16) implies that $|G(z)| \leqslant 1$ for each $z \in \mathbb{C}_{+}^{m}$, moreover,

$$
\left|S_{a}(x+i)\right|^{q}=\left|\exp i \sum_{j=1}^{n} a_{j}\left(x_{j}+i\right)\right|^{q}=\exp \left(-q \sum_{j=1}^{n} a_{j}\right) \leqslant 1 .
$$


Letting $A=\exp \left(-q \sum_{j=1}^{n} a_{j}\right)$, by (20) we obtain that

$$
\left|f_{k}(\widetilde{x+i})\right|^{q}|G(x+i)| \leqslant 2 A,
$$

i.e.,

$$
\left|f_{k}(\widetilde{x+i})\right||G(x+i)|^{\frac{1}{q}} \leqslant(2 A)^{\frac{1}{q}} .
$$

Since the function

$$
F_{k}(z)=f_{k}(\tilde{z}+i)(G(z+i))^{\frac{1}{q}}, k=1,2, \ldots,
$$

can be represented by the Poisson integral (see [13], [14]) in the half-space $\mathbb{C}_{+}^{m}$ and $F_{k} \in$ $H^{\infty}\left(\mathbb{C}_{+}^{m}\right)$, we obtain estimate $(21)$ in half-space $\mathbb{C}_{+}^{m}$, i.e.,

$$
\left|f_{k}(\tilde{z}+i)\right|^{q}|G(z+i)| \leqslant 2 A,
$$

for each $z=\left(z_{1}, \ldots, z_{m}\right) \in \mathbb{C}_{+}^{m}$.

Taking into consideration that

$$
\lim _{k \rightarrow+\infty} f_{k}\left(z_{1}, \ldots, z_{m}, i, \ldots, i\right)=e^{-\sum_{j=1}^{m} i a_{j} z_{j}+\sum_{j=m+1}^{n} a_{j}}, \quad\left(z_{1}, \ldots, z_{m}\right) \in \mathbb{C}_{+}^{m},
$$

and passing to the limit in inequality (22), we finally obtain

$$
\left(\exp \sum_{j=1}^{m} q a_{j} y_{j}+q \sum_{j=m+1}^{n} a_{j}\right) \leqslant 2 A \prod_{j=1}^{m} \exp \left(c p_{j}\left(3\left|z_{j}\right|\right)\right), \quad\left(z_{1}, \ldots, z_{m}\right) \in \mathbb{C}_{+}^{m} .
$$

It follows from (3) that

$$
\lim _{y \rightarrow+\infty} \frac{p_{j}(3 y)}{y}=0, \quad j=\overline{1, m}
$$

But it is impossible in view of estimate (23). The proof is complete.

Proof of Theorem 2. We first prove that if $f \in H^{\infty}\left(\mathbb{C}_{-\eta}^{n}\right), f(z) \neq 0, z \in \mathbb{C}_{+}^{n} \cup \mathbb{R}^{n}$, for some $\eta>0$, then the function $(\ln f)^{m}$ with the principal branch of the logarithm belongs to class $A_{P}^{q}\left(\mathbb{C}_{+}^{n}\right), 1 \leqslant q<+\infty, P \in \Omega$. Indeed, without loss of generality we can assume that $|f(z)| \leqslant 1, z \in \mathbb{C}_{-\eta}^{n}$. Hence, function $\Psi(z)=-i \ln f(z-i \delta)$ satisfies condition $\operatorname{Im} \Psi(z) \geqslant 0$, and $\Psi \in H\left(\mathbb{C}_{\frac{-\eta}{2}}^{n}\right)$. We let $\delta=\frac{\eta}{2}$ and apply Schwarz kind formulae for function $\Psi$ in $\mathbb{C}_{+}^{n}$ (see [13]). We obtain

$$
\Psi(z)=\frac{2 i^{n}}{(2 \pi)^{n}} \int_{R^{n}} \prod_{j=1}^{n}\left(\frac{i+z_{j}}{z_{j}-t_{j}}\right) \frac{1}{i+t_{j}} \times \ln \frac{1}{|f(t)|} d t+i \arg f(i) .
$$

Hence,

$$
|\ln f| z-i \delta|| \leqslant \frac{2}{(2 \pi)^{n}} \int_{R^{n}} \prod_{j=1}^{n}\left(\left|\frac{i+z_{j}}{z_{j}-t_{j}}\right| \frac{1}{i+t_{j}}\right) \times \ln \frac{1}{|f(t-i \delta)|} d t+c_{0} .
$$

Employing the elementary estimate

$$
\sup _{t \in R}\left|\frac{i-t}{z-t}\right|=\frac{|z-i|+|z+i|}{2 \operatorname{Im} z}
$$


where $z \in \mathbb{C}_{+}$(see [12]), we obtain

$$
\begin{aligned}
|\ln | f(z-i \delta)|| & \leqslant \frac{2}{(2 \pi)^{n}} \int_{R^{n}} \prod_{j=1}^{n}\left(\frac{\left|i+z_{j}\right|}{\left|i-t_{j}\right|} \frac{\left|i-t_{j}\right|}{\left|z_{j}-t_{j}\right|\left(i+t_{j}\right)}\right) \times \ln \frac{1}{|f(t-i \delta)|} \\
& \leqslant \frac{2}{(2 \pi)^{n}} \int_{R^{n}} \prod_{j=1}^{n}\left(1+\left|z_{j}\right|\right) \int \frac{\ln \frac{1}{|f(t-i \delta)|}}{\prod_{R^{n}}^{n}\left(1+t_{j}^{2}\right)} \times \prod_{j=1}^{n} \sup _{t_{j} \in R} \mid \frac{i-t_{j}}{z_{j}-t_{j} \mid} \\
& \leqslant \frac{2}{(2 \pi)^{n}} \prod_{j=1}^{n}\left[\left(1+\left|z_{j}\right|\right)\left(\frac{\left|z_{j}-1\right|+\left|z_{j}+1\right|}{2 \operatorname{Im} z_{j}}\right)\right] \times \int_{R^{n}} \frac{\ln \frac{1}{|f(t-i \delta)|}}{\prod_{j=1}^{n}\left(1+t_{j}^{2}\right)} d t_{1} \ldots d t_{n},
\end{aligned}
$$

$t=\left(t_{1}, \ldots, t_{n}\right)$. Thus, we finally get

$$
|\ln | f(z-i \delta)|| \leqslant \text { const } \int_{R^{n}} \frac{\ln \frac{1}{|f(t-i \delta)|}}{\prod_{j=1}^{n}\left(1+t_{j}^{2}\right)} d t \times \prod_{j=1}^{n} \frac{\left(1+\left|z_{j}\right|^{2}\right)}{\operatorname{Im} z_{j}} \leqslant \text { const } \prod_{j=1}^{n} \frac{\left(1+\left|z_{j}\right|^{2}\right)}{\operatorname{Im} z_{j}} .
$$

We let $\zeta=z-i \delta$ in this inequality. If $\operatorname{Im} \zeta_{j} \geqslant 0$, then $\operatorname{Im} z_{j} \geqslant \delta, 1 \leqslant j \leqslant n$. Therefore,

$$
|\ln | f(\zeta)|| \leqslant \text { const } \prod_{j=1}^{n} \frac{\left(1+\left|z_{j}\right|^{2}\right)}{\operatorname{Im} z_{j}} \leqslant \text { const } \prod_{k=1}^{n} \frac{\left(1+\left|z_{k}\right|^{2}\right)}{\delta}, \zeta \in \mathbb{C}_{+}^{n}
$$

Here we have employed the estimate [13]

$$
\int_{R^{n}} \frac{\ln \frac{1}{|f(t-i \delta)|}}{\prod_{j=1}^{n}\left(1+t_{j}^{2}\right)} d t_{1} \ldots d t_{n}<+\infty, \quad t=\left(t_{1}, \ldots, t_{n}\right) .
$$

By estimate $(24)$ we obtain that the function $\Psi_{m}(z)=(\ln f(z))^{m}$ belongs to class $A_{P}^{q}\left(\mathbb{C}_{+}^{n}\right)$ for each $1 \leqslant q<+\infty$.

Now we follow the lines of the proof of Theorem 1 . Let $1 \leqslant q<+\infty$.

We let once again

$$
e(t)=\int_{\mathbb{C}_{+}^{n}} f^{t}(\zeta) \Psi(\zeta) e^{-P(|\zeta|)} d m_{2 n}(\zeta), 0 \leqslant t \leqslant 1
$$

where the principal branch of the power function is used, and $\Psi$ is an arbitrary function in $A_{P}^{q^{\prime}}\left(\mathbb{C}_{+}^{n}\right), \frac{1}{q}+\frac{1}{q^{\prime}}=1$, and

$$
\int_{\mathbb{C}_{+}^{n}} \Psi(\zeta) F(\zeta) e^{-P(|\zeta|)} d m_{2 n}(\zeta)=0
$$

for arbitrary $F \in E_{q}(f)$. We recall that $E_{q}(f)$ is the closure of set $H^{\infty}\left(\mathbb{C}_{+}^{n}\right) f$ in space $A_{P}^{q}\left(\mathbb{C}_{+}^{n}\right)$. It is clear that

$$
e^{(m)}(t)=\int_{\mathbb{C}_{+}^{n}} f^{t}(\zeta)(\ln f(\zeta))^{m} \Psi(\zeta) e^{-P(|\zeta|)} d m_{2 n}(\zeta) .
$$


As in the proof of Theorem 1, let us prove that $e^{(m)}(1)=0, m=0,1 \ldots$ Indeed,

$$
e^{(m)}(1)=\int_{\mathbb{C}_{+}^{n}} f(\zeta)(\ln f(\zeta))^{m} e^{-P(|\zeta|)} d m_{2 n}(\zeta) .
$$

Hence, for an arbitrary sequence $\left\{f_{k}\right\} \in H^{\infty}\left(\mathbb{C}_{+}^{n}\right)$ we have

$$
\left\|f_{k} f-f \Psi_{m}\right\|_{A_{P}^{q}\left(\mathbb{C}_{+}^{n}\right)} \leqslant\|f\|_{\infty}\left\|f_{k}-\Psi_{m}\right\|_{A_{P}^{q}\left(\mathbb{C}_{+}^{n}\right)},
$$

where $\Psi_{m}=(\ln f)^{m}, m \in \mathbb{Z}^{+}$. As it was established above, $\Psi_{m} \in A_{P}^{q}\left(\mathbb{C}_{+}^{n}\right)$, and this is why we can choose a sequence $\left\{f_{k}\right\}^{\infty} \in H^{\infty}\left(\mathbb{C}_{+}^{n}\right)$ such that $\left\|f_{k}-\Psi_{m}\right\|_{A_{P}^{q}\left(\mathbb{C}_{+}^{n}\right)} \rightarrow 0$ as $k \rightarrow+\infty$, $m=1,2 \ldots$

Thus, $f(\ln f)^{m} \in E_{q}(f)$. We proceed to the estimate $e^{(m)}(t)$ on the segment $[0,1]$.

We have

$$
\left|e^{(m)}(t)\right| \leqslant \int_{\mathbb{C}_{+}^{n}}\left|f^{t}(\zeta)\right||\ln f(\zeta)|^{m}|\Psi(\zeta)| e^{-P(|\zeta|)} d m_{2 n}(\zeta) .
$$

Now we employ the estimate

$$
\left|f^{t}(\zeta)\right| \leqslant(|f(\zeta)|+1) \leqslant 2, \zeta \in \mathbb{C}_{+}^{n}, t \in[0,1] .
$$

Then

$$
\left|e^{(m)}(t)\right| \leqslant 2 \int_{\mathbb{C}_{+}^{n}}|\ln f(\zeta)|^{m}|\Psi(\zeta)| e^{-P(|\zeta|)} d m_{2 n}(\zeta) .
$$

Applying Hölder inequality, we arrive at the estimate

$$
\left|e^{(m)}(t)\right| \leqslant 2\left(\int_{\mathbb{C}_{+}^{n}}|\ln f(\zeta)|^{q m} e^{-q P(|\zeta|)} d m_{2 n}(\zeta)\right)^{\frac{1}{q}}\left(\int_{\mathbb{C}_{+}^{n}}|\Psi(\zeta)|^{q^{\prime}} e^{-q^{\prime} P(|\zeta|)} d m_{2 n}(\zeta)\right)^{\frac{1}{q^{\prime}}} .
$$

Therefore, if $0<s<1$, then

$$
\begin{aligned}
\left|e^{(m)}(t)\right| \leqslant & 2\left(\int_{\mathbb{C}_{+}^{n}}\left(|\ln f(\zeta)|^{m} e^{-s P(|\zeta|)}\right)^{q} e^{(-q(1-s) P(|\zeta|))} d m_{2 n}(\zeta)\right)^{\frac{1}{q}} \\
& \cdot\left(\int_{\mathbb{C}_{+}^{n}}|\Psi(\zeta)|^{q^{\prime}} e^{-q^{\prime} P(|\zeta|)} d m_{2 n}(\zeta)\right)^{\frac{1}{q^{\prime}}} \\
\leqslant & 2 M_{m}\left(\int_{\mathbb{C}_{+}^{n}} e^{-(q(1-s) P(|\zeta|))} d m_{2 n}(\zeta)\right)^{\frac{1}{q}}\left(\int_{\mathbb{C}_{+}^{n}}|\Psi(\zeta)|^{q^{\prime}} e^{-q^{\prime} P(|\zeta|)} d m_{2 n}(\zeta)\right)^{\frac{1}{q^{\prime}}}
\end{aligned}
$$

where $s \in(0,1)$. In view of $P \in \Omega$ we finally we obtain

$$
\left|e^{(m)}(t)\right| \leqslant A^{m} M_{m}, \quad m \in \mathbb{Z}_{+}, \quad t \in[0,1] .
$$

Now we employ the condition $e^{(m)}(1)=0, m=0,1, \ldots$ At that, the convergence of the series implies that $e$ belongs to Carleman-Ostrowski quasi-analytic class (see [12]). Hence, $e(0)=0$. The proof is complete. 


\section{BIBLIOGRAPHY}

1. N.K. Nikolski. Operators, functions and system: An easy reading. V. 1. Hardy, Hankel, and Toeplitz. Math. Surveys Monograph. 92. Amer. Math. Soc., Providence, RI (2002).

2. M.V. Keldysh. Sur l'approximation en moyenne par polynômes les functions d'une variable complexe // Matem. Sborn. 16:1, 1-20 (1945).

3. A. Beurling. A critical topology in harmonic analysis on semigroups // Acta. Math. 112:3-4, 213-215 (1964).

4. N.K. Nikol'skii. Selected problems of weighted approximation and spectral analysis // Trudy Mat. Inst. Steklov. 120, 3-272 (1974). [Proc. Steklov Inst. Math. 120:1-276 (1974).]

5. I.M. Gevorkyan, F.A. Shamoyan. On weak invertibility in spaces of functions analytic in the disk admitting growth close to its boundary // Dokl. Akad. Arm. SSR. 82:4, 156-159 (1986). (in Russian).

6. O. El-Fallah, K. Kellay, K. Seip. Cyclicity of singular inner functions from the corona theorem // J. Inst. Math. Jussieu. 11:6, 815-824 (2012).

7. J.B. Garnett. Bounded analytic functions. Pure and Applied Mathematics, 96. Academic Press, New York (1981).

8. F.A. Shamoyan. Weakly invertible elements in anisotropic weighted spaces of holomorphic functions in a polydisc // Matem. Sborn. 193:6, 143-161 (2002). [Sb. Math. 193:6, 925-943 (2002).]

9. M.M. Dzhrbashyan. Metric tests of completeness of a system of polynomials under a weighted approximation // Dokl. Akad. Nauk SSSR. 56:6, 1034-1040 (1949). (in Russian).

10. S.N. Mergelyan. Weighted approximations by polynomials // Uspekhi Matem. Nauk. 9:5, 107-152 (1956). (in Russian).

11. F.A. Shamoyan. Weak invertibility in weight spaces of analytic functions // Izv. RAN. Ser. Matem. 60:5, 191-212 (1996). [Izv. Math. 60:5, 1061-1082 (1996).]

12. P. Koosis. The Logarithmic integral, I. Cambridge Univ. Press, Cambridge (2004).

13. V.S. Vladimirov. Generalized functions in mathematical physics. Nauka, Moscow (1979). [Mir Publishers, Moscow (1979).]

14. E.M. Stein, G. Weiss. Introduction to Fourier analysis on Euclidean spaces. Princeton Mathematical Series. Princeton Univ. Press, Princeton (1971).

Faizo Agitovich Shamoyan,

Research laboratory of complex

and functional analysis,

Bryansk State University named

after Academician I.G. Petrovsky,

Bezhitskaya str., 14,

241036, Bryansk, Russia

E-mail: shamoyanfa@yandex.ru 